\title{
MECHANISMS AND CONTROL OF MACROSEGREGATION IN DC CASTING
}

\author{
Dmitry G. Eskin \\ Brunel University, Brunel Centre for Advanced Solidification Technology; Kingston Lane; Uxbridge; UB8 3PH, United Kingdom
}

Keywords: Macrosegregation, DC casting; Grain refinement; Floating grains; Convection; Shrinkage

\begin{abstract}
Macrosegregation is a severe, unrecoverable defect often occurring in large-scale castings. This paper offers a critical review of mechanisms involved in the formation of macrosegregation during DC casting of aluminum alloys. These mechanisms include thermo-solutal and forced convection, shrinkage-driven flow and transport of solid crystals. It is demonstrated that the impact of melt flow on macrosegregation depends on the flow direction and pattern, and on the extent of the slurry zone in the sump. The shrinkage-induced flow contributes to the negative centerline segregation but this contribution depends on the shape of the macroscopic solidification front and on the permeability of the mushy zone. Accumulation of floating crystals will result in negative segregation but the occurrence of these grains and their composition depends on grain refinement and melt flow pattern. It is shown that macrosegregation can be controlled by practical means such as process parameters, structure modification and melt flow management.
\end{abstract}

\section{Introduction}

The fact that large-scale castings and ingots are not homogeneous with respect to their chemical composition has been known for centuries. It is widely cited that Italian metallurgist and foundryman $\mathrm{V}$. Biringuccio described segregation in bronze gun barrels in his book "De la Pirotechnia" as early as in 1540. In 1574 Austro-Hungarian chemist L. Ercker published his observations of liquation in precious alloys. Most observations and studies of macrosegregation during the 19-th century has been done on precious metals, including works by W.C. Roberts-Austin (1875) and E. Matthey (1890) in Great Britain. It is acclaimed that Russian metallurgists A.S. Lavrov and N.V. Kalakutsky in 18661867 observed macrosegregation in steel ingots and noted that its degree depended on the size of the ingot. Lavrov wrote that the cause of macrosegregation was in the precipitation of carbon during steel solidification and accumulation of low-melting components in the center of the ingot. It was not until the beginning of the XX-th century, however, that the macrosegregation attracted real scientific interest, first as related to steel and bronze ingots and, later - to aluminum billets and ingots. We can mention the pioneering works of T. Turner, M.T. Murray, E.A. Smith, O. Bauer, H. Arndt, R.C. Reader, R. Kühnel, and F.W. Rowe in copper alloys and those of G. Masing, W. Claus, S.M. Voronov, and W. Roth in aluminum alloys (citation information is available in Ref. [1]).

During the first half of the XX-th century quite a number of theories have been suggested for the explanation of inverse segregation. These theories attempted to explain the numerous observations, some of which are summarized here. It was experimentally found that the inverse segregation occurred in alloys with a considerable freezing range and the extent of the segregation increased with the freezing range, e.g. works by Claus and Goederitz in 1928 and Voronov in 1927 and 1929. The presence of hydrogen was shown to promote exudations, while melt overheating increased the degree of segregation. Generally it was concluded by Masing and Dahl as early as in 1926 that hydrogen in aluminum alloys would adversely affect macrosegregation if it were trapped in the mushy zone. Therefore this influence was only typical of moderate cooling rates, when hydrogen was neither quenched in solid aluminum nor escaped the solidifying metal. The cooling rate was noted to be a determining factor in segregation already in early accounts, e.g. J.T. Smith in 1875. Bauer and Arndt (1921) emphasized a steep temperature gradient in the ingot as an essential condition for macrosegregation. Voronov (1929) showed that any change in casting conditions which increased the cooling rate, i.e. reduced casting temperature, colder mold, lower pouring rate, increased mold conductivity, would increase the degree of inverse segregation in duralumin ingots.

The properties and grain structure of an alloy were also under scrutiny in relation to macrosegregation. First of all it was shown that the segregation developed during solidification and not in the liquid state as has been thought until the 1920s. The transition from the normal to inverse segregation was experimentally observed on increasing the thickness of the solidified shell of an ingot, e.g. by Fraenkel and Gödecke in 1929. The inverse segregation was often less in finer equiaxed structures than in columnar or coarse dendritic structures. This was related to the different mechanisms of feeding the solidification contraction, i.e. liquid feeding in columnar structures and mass feeding in equiaxed structure. As early as in 1925, Masing et al. correlated inverse segregation to volume contraction during solidification of metallic alloys. This theory was further developed by Phelps (1926) and Verö (1936) and formed a basis for the modern views on macrosegregation.

As applied to direct-chill casting the macrosegregation theory was developed in Russia based on the experimental studies by V.I. Dobatkin (1948), N.F. Anoshkin (1976) and V.A. Livanov (1977). The role of the formation of the macroscopically continuous and microscopically discontinuous solidification front was emphasized in the works of Dobatkin (1948). In modern terms it would be the ratio between coherency and permeability of the mushy zone. It was experimentally and analytically shown that the degree of macrosegregation is related to the shape of the billet sump and, therefore to the casting speed and the billet size. The transition between positive and negative centerline segregation was shown to be possible in dependence on the shrinkage ratio and the convection development.

The modern theory of macrosegregation was formulated in formalized in the works of M.C. Flemings in the 1960-1970s. This is essentially the modern macrosegregation theory as we know it now. Since that time a lot of efforts was devoted to the development of models for computer simulation of macrosegregation and much less - to the experimental studies. 
Our work was focused on finding the interaction between different macrosegregation mechanisms and on looking on the individual contributions of specific macrosegregation mechanisms.

The basics of all macrosegregation mechanisms can be formulated as the relative movement of liquid and solid phases during solidification [1, 2, 3]. This relative movement translates the partitioning of solute elements between liquid and solid phases (micro-segregation) to the difference of chemical composition on the macroscopic scale (macrosegregation). There are, however, different types of such a relative movement that are characteristic of different parts of the transition region of a casting, e.g. in the sump of a billet during direct-chill casting:

- thermo-solutal convection caused by temperature and concentration gradients, and the penetration of this convective flow into the slurry and mushy zones of a billet (see Fig. 1);

- transport of solid grains within the slurry zone by gravity and buoyancy forces, convective or forced flows;

- melt flow in the mushy zone that feeds solidification shrinkage and thermal contraction during solidification;

- melt flow in the mushy zone caused by metallostatic pressure;

- melt flow in the mushy zone caused by deformation (thermal contraction) of the solid network;

- forced melt flow caused by pouring, gas evolution, stirring, vibration, cavitation, rotation etc., which penetrates into the slurry and mushy zones of a billet or changes the direction of convective flows.

We know that commercial alloys usually solidify as dendrites, forming overall equiaxed structure in a billet. In the slurry zone (between the liquidus and the coherency isotherm in the transition region) the equiaxed grains are free to move and can travel short or long distances, depending on their size and direction of melt flow (see Fig. 1). In the mushy zone (between the coherency isotherm and the nonequilibrium solidus) however, these dendrites form a continuous solid network and have a fixed position in the billet. It can be considered that they move only in the direction of billet withdrawal and with the casting speed. Liquid flow within the mushy zone is limited to distances comparable to several grain sizes.

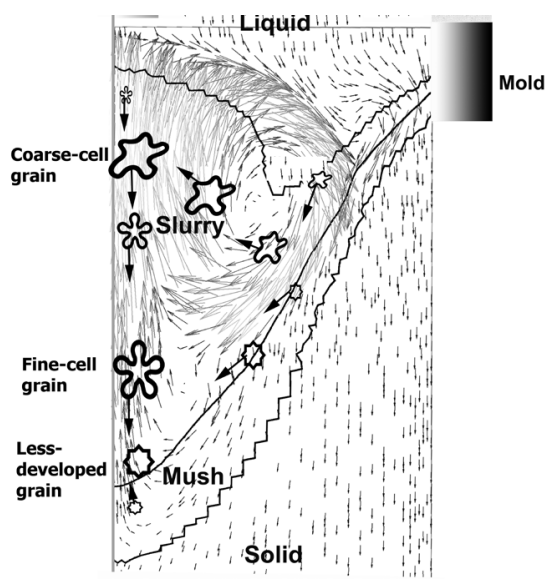

Figure 1. A scheme showing the typical melt flow pattern in the transition region of the sump of a DC cast round billet with liquidus, coherency and solidus isotherm separating liquid, slurry, mush and solid parts of the billet. Possible trajectories of free-floating crystals are shown. Only half of the billet is shown with the centerline on the left.

\section{Convection-driven macrosegregation}

One of the most recognized phenomena behind macrosegregation is thermo-solutal convection, or the melt flow driven by temperature and concentration gradients. These gradients exist in the liquid (or more correctly - fluid) part of a casting (billet) due to uneven cooling of the whole volume. The typical convective flow pattern in a DC cast billet is shown in Fig. 1. The main reason why this flow may affect the distribution of alloying elements in the billet cross-section is the penetration of this flow into the slurry zone and washing out of the liquid with the composition already changed by the solidification process. The interaction between the liquid pool and the transition zone of the billet was noted as the main reason for convection-driven segregation by Tageev in 1949 [4]. In addition, the thermo-solutal flow may assist in transporting the solid phase within the slurry region and to the liquid pool (see Fig. 1).

Figure 1 shows that the penetration of the melt flow into the slurry zone occurs in the outer quarter of the billet cross-section. Thus the solute-enriched liquid from this part of the billet is mixed with the bulk liquid and the resultant mixture is brought to the centre of the billet. The result is centerline positive segregation as shown in Fig. 2 a by curve 1 . At the billet periphery the melt flow is directed towards the surface (Fig. 1). Here the liquid of the nominal composition penetrates the mushy zone and dilutes the melt that is enriched there by solidification. Hence, a negative segregation at the billet periphery is facilitated (curve 1 in Fig. 2a). Generally, we can conclude that the natural thermo-solutal convection in DC cast billets of aluminum alloys enhances the normal (direct) macrosegregation.

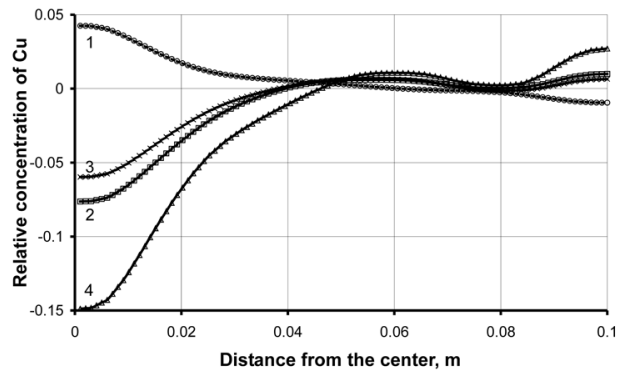

a

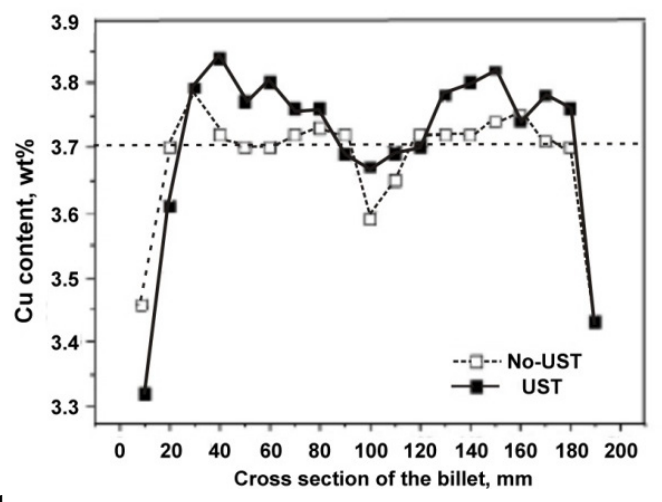

b

Figure 2. (a) Computer simulation of macrosegregation in a DC cast billet of an $\mathrm{Al}-\mathrm{Cu}$ alloy with only thermo-solutal convection (1); with thermo-solutal convection and shrinkageinduced flow (2); same as 2 but with permeability of the mush increased two times (3); same as 2 but with the Scheil model of solidification (4) [5] and (b) experimental results on macrosegregation upon DC casting of a $200-\mathrm{mm}$ billet with forced centerline downward flow induced by ultrasonic horn in the hot top. 
It has been clearly demonstrated experimentally that the macrosegregation pattern depends on the extent and direction of convection [6]. The downward centerline flow enhances the positive centerline segregation by forcing the enriched liquid to stay and solidify in the centre; whereas the upward centerline flow facilitates negative centerline segregation, extracting the enriched liquid from the transition region. This is illustrated in Fig. 3 where the results of experiments and computer simulations are given together. In this case a mechanical pump was placed in the liquid part of the sump of the billet. Other means of forced convection may lead to similar results. As an example, Fig. $2 b$ shows the effect of the downward forced flow induced by the ultrasonic sonotrode (horn) placed in the hot top along the centerline of the billet. Macrosegregation pattern changes with the same trend as with the pump, i.e. the extent of negative centerline segregation becomes less. Effectively, one can control the extent and mode of macrosegregation by controlling the direction of melt flow in the sump, providing all other conditions remain the same.

\section{Shrinkage-driven macrosegregation}

Historical accounts summarized elsewhere [1, 3] show that the importance of shrinkage-driven flows for the formation of inverse segregation has been realized as early as in the 1930s. The inverse segregation is caused by the movement of the solute-rich liquid in the direction opposite to the movement of the solidification front. In the case of DC casting, that would be a melt flow directed from the centre to the periphery of a billet (ingot).
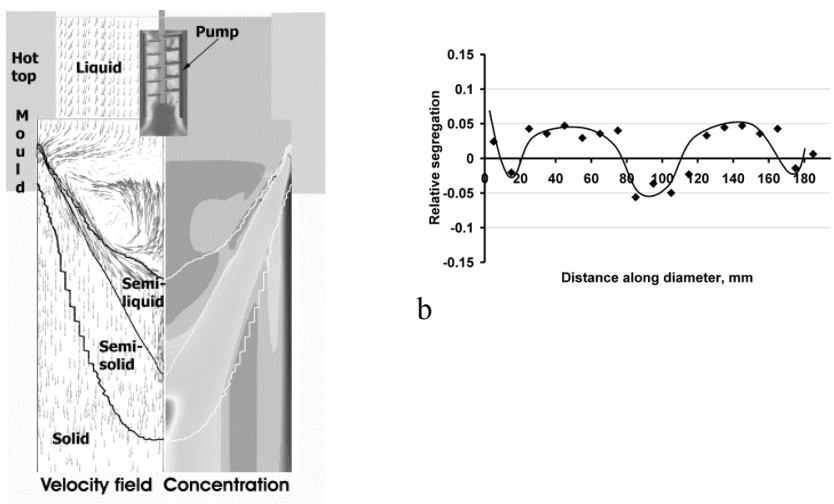

a
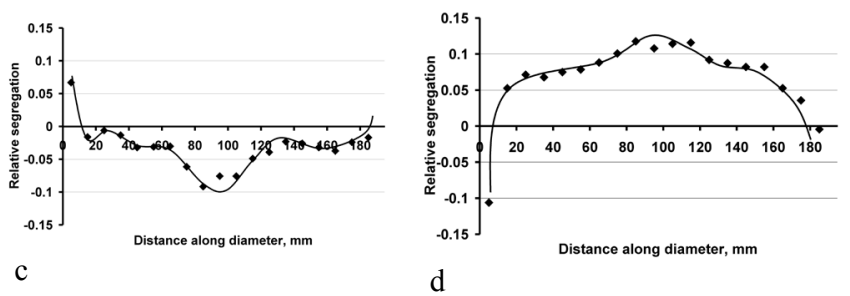

Figure 3. Effect of forced centerline convection induced by a mechanical pump on the extent and direction of macrosegregation upon DC casting of a binary Al-Cu alloy: (a) position of the pump; (b) natural convection; (c) upward forced flow; (d) downward forced flow [7].

Solidification shrinkage is a result of density change during solidification and occurs throughout the entire solidification range. In the slurry zone, however, the solidification shrinkage is easily compensated by the melt flow. There is no pressure difference that may result in the additional flows and the solidification shrinkage does not play any significant role in the relative movement of solid and liquid in the slurry zone, the main influence being exerted by thermo-solutal convection. Deeper into the mushy zone when the permeability is limited and the feeding of the solid phase is restricted, the solidification shrinkage (assisted closer to the solidus by thermal contraction of the solid phase) causes the pressure difference over the solidifying layer of the mushy zone that creates the driving force for the so-called "shrinkage-driven" flow. The flow in the mushy zone, in spite of its small magnitude, involves highly enriched liquid, which determines its significance for the macrosegregation. It is important that this flow is directed perpendicular to the solidification front.

The horizontal component of shrinkage-induced flow velocity vector takes the solute away from the centre to the surface, though this solute transport physically occurs very slowly. Step by step, however, an overall solute transfer occurs from the centre of the billet to its surface. The depletion in the centre cannot be compensated, as there is no horizontal inflow of the solute from more enriched regions. At the surface, there is a pile-up of the solute as there is no outflow. Because the magnitude of the shrinkage-induced flow is dependent on the shrinkage ratio, one may conclude that the corresponding macrosegregation should depend on the dimensions of the mushy zone and the degree of shrinkage. There is also a clear correlation between the shape of the solidification front and the degree of shrinkage-induced segregation, which has been noted already in the 1940s [8]. The deeper the sump, the more solute is taken from the centre of the billet to the periphery [9].

By taking solidification shrinkage and solidification front inclination into account, the total amount of the transferred solute at different positions during the solidification period $\left(L_{\mathrm{m}} / V_{\text {cast }}\right)$ is as follows [9]

$$
L_{\mathrm{h}}=\int_{0}^{L_{M} / V_{\text {cast }}} C_{1} f_{1} V_{\text {cast }} \beta(\sin 2 \alpha) / 2 d t,
$$

where $V_{\text {cast }}$ is the casting speed; $\beta$ is shrinkage ratio ( 0.1 for an aluminum alloy); $\alpha$ is the local slope of the coherency isotherm; $L_{\mathrm{m}}$ is the vertical thickness of the mushy zone; $C_{1}$ is liquid phase concentration; and $f_{1}$ is the volume fraction of liquid phase. This equation can be reduced for $\mathrm{Cu}$ (partition coefficient $\mathrm{K}_{\mathrm{Cu}}$ is taken as 0.171$)$ as [9]

$$
L_{\mathrm{h}}=0.78 C_{0} L_{M} \beta(\sin 2 \alpha) / 2,
$$

The derivative of Eq. (2) $\mathrm{d} L_{\mathrm{h}} / \mathrm{d} r$ ( $r$ is the radial distance from the billet center) is the net efflux and is a measure of the macrosegregation caused by solidification shrinkage.

Hence, there is a direct link to the shape of the sump and to the casting speed.

Computer simulations using a model that includes solidification shrinkage demonstrate the high potential of the shrinkage-driven flow in the formation of inverse segregation during DC casting [9]. Figure 2a (curves 2-4) gives a clear evidence of that. Note also the effect of permeability that is the function of structure. We can conclude that the shrinkage-induced flow is responsible for the occurrence of negative centerline segregation. 


\section{Free-floating grains and macrosegregation}

Movement and sedimentation/growth of solid grains in the slurry zone is sometimes considered as the main mechanism of centerline segregation in DC cast billets and ingots $[10,11]$. At the same time, as we discussed in the previous section, the negative centerline segregation can be explained by the action of the shrinkage-induced flow. Frequent occurrence of duplex grain structures in the center of a billet (grains with coarse and fine internal structure) is taken as an evidence of solid-phase transport (floating) within the transition region (see Fig. 1), which might affect the extent of the centerline segregation. The mainstream concept assumes that if the coarse-cell dendrites are solute-poor, fine dendrites (the last to solidify) are rich in solute [10, 12].

If we assume that floating grains arrive from other part of the billet and settle in its centre, then they effectively bring there more solid phase than it should be at this point at time and space. As the primary solid in hypoeutectic aluminum alloys is always depleted of the solute, the accumulation of the floating grains has to contribute to the negative segregation.

Electron-probe microanalysis (EPMA) measurements on several coarse and fine cells in the duplex structures found in the centre of 195-mm billets from 2024 and 7075 alloys clearly demonstrate that coarser cells are more solute-depleted as compared to the 'regular' finer cells [13, 14]. Both grain-refined (GR) and nongrain refined (NGR) alloys were tested. Figure 4 gives some illustrations. In the case of grain refining, the difference in minimum concentrations is less, probably due to a more efficient back diffusion in the finer structure. The areas occupied by finecell grains are close by composition to the average alloy composition or are enriched in solutes, especially in GR alloys cast at a low speed. From these measurements, the minimum $\mathrm{Cu}$, $\mathrm{Mg}$, and $\mathrm{Zn}$ concentrations in the centre of dendrite cells were tabulated. These values were then compared with the composition of the aluminum solid solution in equilibrium with the liquid during solidification and the corresponding volume fraction of solid was derived based on the Scheil solidification model using Thermocalc. The results clearly demonstrate that coarse-cell grains, fine-cell grains and less developed dendrites have been formed in different parts of the sump as depicted in Fig. 1.

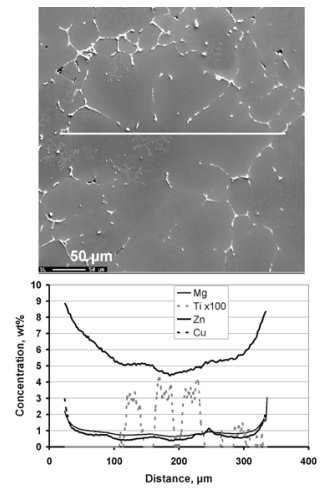

a

Figure 4: Distribution of alloying elements in (a) coarse-cell and (b) fine-cell grains in the center of a grain refined 7075 alloy

The obtained results and their analysis allow us to make the following conclusions. There are two mechanisms contributing to the negative centerline segregation: shrinkage-induced flow and free-floating grains. It is important to realize that the shrinkageinduced flow is a physical phenomenon that is always present in the transition region of a solidifying billet, whereas the transport of solute-lean solid with its accumulation in a certain part of a casting is a conditional incident, the occurrence of which depends on a number of factors such as the structure evolution, temperature regime, and the direction of strong flows.

\section{Control of macrosegregation by a melt distribution system}

The control of melt flow in the sump can be achieved by using different melt distribution systems while feeding the melt into the liquid pool of a billet. Such systems are widely used in DC casting. Two systems were specifically tested macrosegregation response of $315-\mathrm{mm}$ billets from an AA7050 alloy, i.e. a semihorizontal scheme when the melt is directed through four outlets from the centerline to the sides of the billet and a vertical scheme when the melt is directed downward along the centerline. The experimental and computer simulation details are reported elsewhere [15].

The obtained macrosegregation patterns are distinctly different as shown in Fig. 5. While the semi-horizontal scheme produces the macrosegregation typical of DC cast billets, the vertical scheme results in solute enrichment in the center of the billet followed by deep negative segregation troughs.
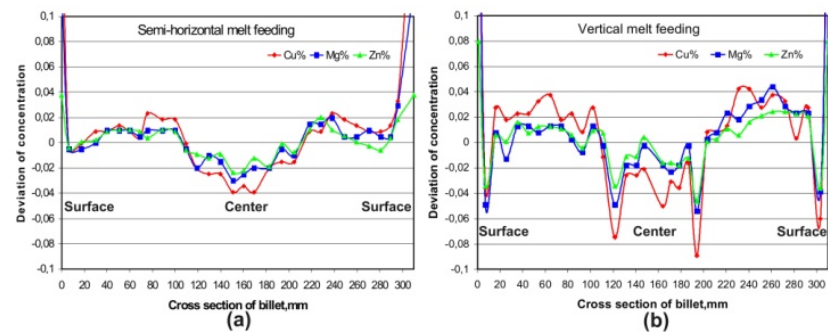

(b)

Figure 5. Relative segregation of alloying elements across the billet diameter: (a) semi-horizontal melt feeding billet; and (b) vertical melt feeding billet.
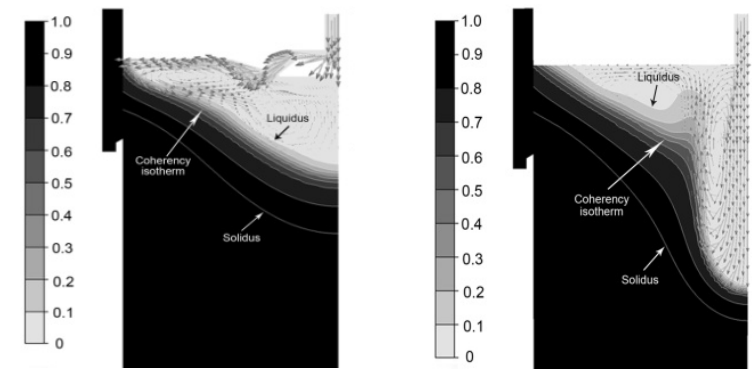

Figure 6: Simulated sump profiles and flow patterns during DC casting: (a) semi-horizontal melt feeding billet, maximal velocity is $15 \mathrm{~mm} / \mathrm{s}$ at $20 \mathrm{~mm}$ from the surface; (b) vertical melt feeding billet, maximal velocity is $30 \mathrm{~mm} / \mathrm{s}$ close to the melt outlet. Centerline is on the left.

In order to understand the formation of such macrosegregation patterns, the structure of the billets was examined and the sump shape and melt flows were simulated [15]. It appears that the vertical feeding scheme results in significant sump depression in the center of the billet with very high flow velocities close to the melt outlet, while for the semi-horizontal scheme the maximum flow velocity occurs close to the billet surface. Figure 6 shows the simulated flow patterns and sump profiles. The uneven change in 
the sump profile and distances between solidus and liquidus in the vertical feeding scheme cause sharp increase in the cooling rate in the center followed by much lower cooling rate at about $40 \mathrm{~mm}$ off center [15]. A combination of melt flow velocity and cooling rate affects the grain structure formed with local grain refinement in the center of the billet in the vertical scheme compared to grain coarsening in the semi-horizontal scheme.

Figure 6a shows that under semi-horizontal feeding, the penetration of melt flow into the slurry zone is more pronounced near the surface area. This means that the effect of washing out of solute-rich liquid from the slurry zone in this area is larger than in the quarter and center of the billet. This solute-rich liquid moves then towards the quarter and central areas along with the convection flow, which results in the lack of alloying elements near the surface area and the positive segregation in the quarter position. In the vertical feeding setting, the flow pattern is completely different. There are two convection flows as illustrated in Fig. 6b. One is near the center area, clockwise. Another is at the quarter and surface of the billet, in the counter-clockwise direction. The two flows collide at the position $40-50 \mathrm{~mm}$ away from the center. The most pronounced washing out of soluteenrich liquid from the slurry zone happens around the cliff-shaped solidification front (40 mm away from the center). This strong upstream flow in the slurry zone might wash out the enriched solute either to the center or to the surface area, which causes negative segregation in this area, as shown in Fig. 5 b.

The movement of solid grains along with convection flow is one of the macrosegregation mechanisms as has been discussed earlier in this paper. Figure 7 gives a hypothetical path of floating crystals movement in the vertical feeding scheme.

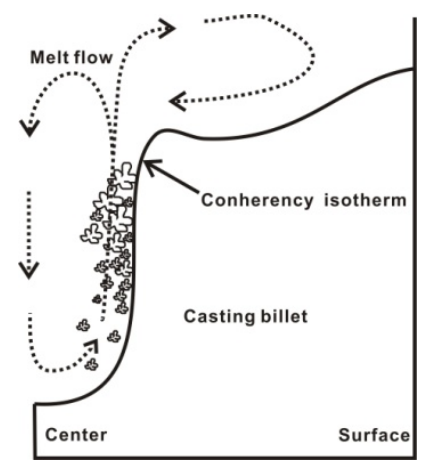

Figure 7. Schematic view of relative movement of melt and grains along cliff-shaped sump in the vertical melt feeding scheme.

A cliff-shaped sump leads to an almost vertical solidification front approximately $40 \mathrm{~mm}$ away from the center. The grains, which are formed in the slurry zone around this vertical solidification front or formed earlier at other position before arriving in this area with melt flow, will stack and grow at the same position of almost vertical solidification front because of strong upstream melt flow and gravity. The low cooling and solidification rates in this region further promote the formation of these coarse grains.

In addition to the almost vertical solidification front, this area is also the place where collision occurs between two flow vortices. As a result, there is almost no horizontal velocity component. In this case, solid grains cannot be displaced from that cliff-shaped front due to stack of grains, but solute enriched melt can. Hence, the strong upstream flow will continue to take the solute enriched liquid away from this slurry zone but leave the solute depleted grains. This may further enhance negative segregation in this cliff shaped sump area.
The next mechanism of macrosegregation that acts in the given case is the shrinkage-induced flow. We can use Eqs. (1) and (2) to estimated the extent of shrinkage-induced segregation. If we compare the calculated shrinkage-induced segregation in Fig. $8 \mathrm{a}$ with real $\mathrm{Cu}$ segregation in the semi-horizontal feeding scheme (Fig. 5a), a good agreement can be found [16]. It should be noted that the segregation curve in Fig. 8a reflects only the calculated segregation caused by shrinkage-driven flow. As discussed before, the movement of solute-enrich liquid in the slurry zone from the surface to the center contributes to a centerline positive segregation. Taking this into account, negative 6.5 rel.\% deviation of $\mathrm{Cu}$ in the center caused by the shrinkage flow (Fig. 8a) might be compensated by the effect of convection flow in the slurry zone. As a result, the negative centerline segregation in the real cast billet reduces to about 4 rel.\%, as shown in Fig. 5a.

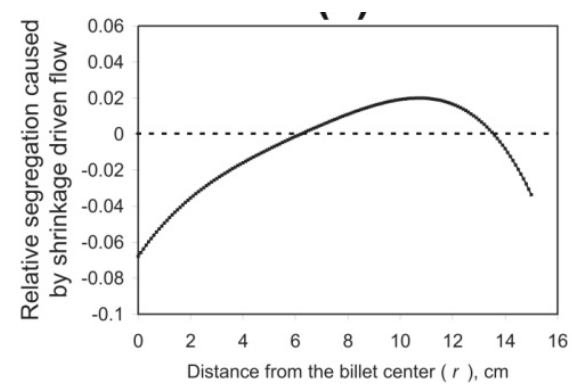

a

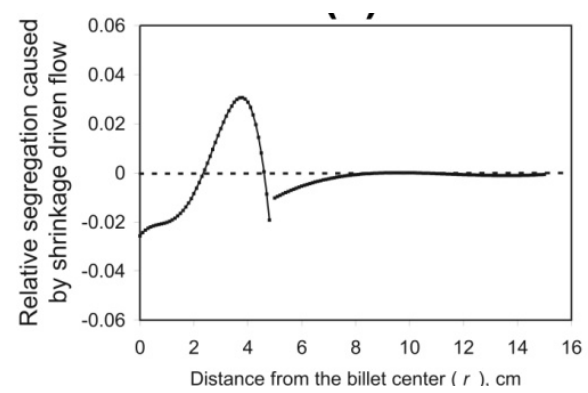

Figure 8. Estimated shrinkage-induced segregation for two melt feeding schemes: (a) semi-horizontal and (b) vertical.

Same analysis can be done for the vertical melt feeding scheme. Figure $8 \mathrm{~b}$ shows the relative $\mathrm{Cu}$ segregation from the center to the surface of the billet. The agreement between this segregation pattern and real segregation in Fig. 5b is not as good as in semihorizontal feeding billet. But there is an obvious negative segregation at the position $40-50 \mathrm{~mm}$ away from the center, which shows the shrinkage driven flow might also contribute to the strong off-center negative segregation in addition with the convention flow and "floating" grains as discussed above.

The shrinkage-driven flow also causes a positive segregation at the position 25-40 mm away from the center, as shown in Fig. 8b. Although the positive segregation in this area does not appear in the real segregation curve (Fig. 5b) (only a slight increase in the concentration can be observed), it becomes logical when the melt flow in slurry zone is also considered. This positive segregation area caused by shrinkage-driven flow coincides with the position where a strong upstream flow is. Therefore the liquid near the coherency isotherm could be already depleted by this upstream flow and might not be rich enough to allow the shrinkage-driven flow to create such a positive segregation.

This analysis of complex macrosegregation patterns observed in DC cast billets demonstrates how different mechanisms of 
macrosegregation act together or counteract each other. It is obvious that the control of melt flow does not only act as a component of convection in the liquid part of the billet sump but also affect other mechanisms of segregation through changing the sump geometry and the trajectory of floating grains. Application of melt distribution systems, ultrasonic processing or specially designed pumps can be efficiently used for the control of macrosegregation patterns in DC casting.

\section{Scaling of macrosegregation}

The interplay of different mechanisms of macrosegregation can result in very different macrosegregation patterns that can be understood and explained only when the whole plethora of phenomena is taken into account. The flow velocities and directions; the easiness of floating and settling of free grains; the shape of the sump; the ratio between slurry and mushy zones; he permeability of the transition regions; and the grain refinement all play their role in different mechanisms and determine the contribution of these mechanisms. In principle, any macrosegregation pattern can be achieved and observed in DC casting, including negligible segregation. This is illustrated in Fig. 9 as a scaling plot [17]. The process parameters such as billet diameter and casting speed determine the flow pattern and the sump shape, while grain refinement affects floating grains and permeability of the transition region. The lines show the regimens when negligible macrosegregation can be achieved.

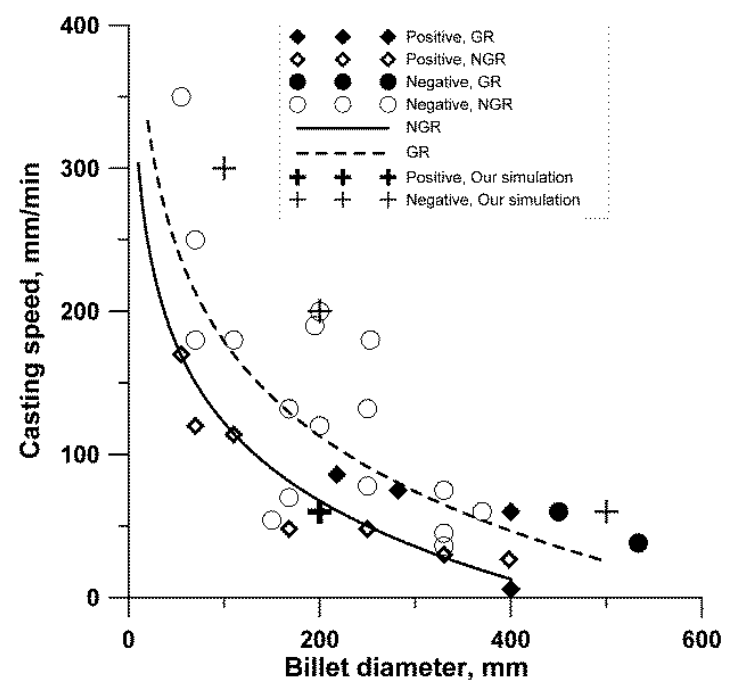

Figure 9. Effect of billet diameter and casting speed on the sign of centerline segregation upon DC casting of various aluminum alloys. Dashed line is for grain refined (GR) and solid line - for not grain-refined alloys (NGR).

\section{Conclusions}

Experimental studies together with computer simulations enable one to study the contributions of different mechanisms into the overall macrosegregation observed during DC casting of aluminum alloys. Computer simulation gives a unique opportunity to inspect the separate contributions of different mechanisms that are practically inseparable in experiment. On the other hand carefully designed experiments can shed a light on the mechanisms that are yet to be reliably modeled. Convection affects the segregation pattern in dependence on the flow direction. Natural convection in the sump of a DC cast billet enhances the positive centerline segregation. Shrinkage flow facilitates the negative centerline segregation. There is a direct correlation between the geometry of the billet sump (affected by the process parameters) and the degree of macrosegregation. The transport of solid grains in the sump of a DC cast billet contributes to the negative centerline inverse segregation. The overall macrosegregation pattern observed in real billets and ingots is a result of complex combination of different mechanisms, which is affected by the structure and process parameters. In principle it is possible to obtain positive or negative centerline segregation for a wide range of billet sizes by the control of the casing speed, which determines the shape of the sump and, therefore the extent of shrinkage and convection flows, and the formation and transport of floating grains. This is illustrated in Fig. 9.

\section{Acknowledgements}

The results reported in this paper have been obtained within the framework of research program of Materials innovation institute (www.M2i.nl). Author would like to thank Prof. L. Katgerman (TU Delft), Drs Q. Du, R. Nadella, A.N. Turchin, D. Ruvalcaba, L. Zhang (M2i/TU Delft) for their valuable contribution to results.

\section{References}

[1] D.G. Eskin: Physical Metallurgy of Direct-Chill Casting of Aluminum Alloys (CRC Press, Boca Roton, 2008).

[2] M.C. Flemings: ISIJ Intern. 40 (2000) 833-841.

[3] R. Nadella, D.G. Eskin, Q. Du, and L. Katgerman: Progr. Mater. Sci. 53 (2008) 421-480.

[4] T.M. Tageev: Dokl. Akad. Nauk SSSR 67 (1949) 491-494. [5] Q. Du, D.G. Eskin, L. Katgerman: Metall. Mater. Trans. A 38 (2007) 180-186.

[6] D.G. Eskin, A.N. Turchin, and L. Katgerman: Intern. J. Cast Metals Res. 22 (2009) 99-102.

[7] D.G. Eskin, A. Jafari, and L. Katgerman: Mater. Sci. Technol. 27 (2011) 890-896.

[8] V.I. Dobatkin: Continuous Casting and Casting Properties of Alloys (Oborongiz, Moscow, USSR, 1948) pp. 83-96.

[9] D.G. Eskin, Q. Du, and L. Katgerman: Scr. Mater. 55 (2006) $715-718$

[10] H. Yu and D.A. Granger: (1986) Aluminum Alloys - Their Physical and Mechanical properties, Ed. by E.A. Starke Jr. and T.H. Sanders Jr. (University of Virginia, Charlottesville, 1986) pp. $17-29$.

[11] M.G. Chu and J.E. Jacoby: Light Metals 1990, Ed. by C.M. Bickert (TMS, Warrendale, 1990) pp. 925-930.

[12] R.C. Dorward and D.J. Beerntsen: Light Metals 1990, Ed. by C.M. Bickert (TMS, Warrendale, 1990) pp. 919-924.

[13] D.G. Eskin, R. Nadella, and L. Katgerman: Acta Mater. 56 (2008) 1358-1365.

[14] D.G. Eskin and L. Katgerman: Aluminium Alloys: Their Physical and Mechanical Properties, Ed. by J. Hirsch, B. Skrotzki, and G. Gottstein (Wiley-VCH, Weinheim, 2008) pp. 292-297.

[15] L. Zhang, D. G. Eskin, A. Miroux, T. Subroto, L. Katgerman: Metall. Mater. Trans. B 23B (2012) 1565-1573.

[16] L. Zhang, D.G. Eskin, A. Miroux, T. Subroto, L. Katgerman: IOP Conf. Ser.: Mater. Sci. Eng., 2012, vol. 33, paper 012019. [17] D.G. Eskin, Q. Du, L. Katgerman: Metall. Mater. Trans. A 39A (2008) 1206-1212. 